\title{
A PUZZLE ABOUT THE EXPERIENCE OF LEFT AND RIGHT
}

\author{
Brian Cutter \\ University of Notre Dame
}

\begin{abstract}
Imagine your mirror-inverted counterpart on Mirror Earth, a perfect mirror image of Earth. Would her experiences be the same as yours, or would they be phenomenally mirror-inverted? I argue, first, that her experiences would be phenomenally the same as yours. I then show that this conclusion gives rise to a puzzle, one that I believe pushes us toward some surprising and philosophically significant conclusions about the nature of perception. When you have a typical visual experience as of something to your left, the following three claims seem very plausible: (1) No one could have an experience phenomenally just like yours without thereby having an experience as of something to her left. (2) Your experience is veridical. (3) Your experience doesn't differ from that of your mirror-inverted counterpart with respect to veridicality. But (1)-(3) jointly contradict the claim that you and your mirror-inverted counterpart would have the same experiences. I argue that any viable response to this puzzle will embrace the following disjunction: either there is a degree of independence between perceptual phenomenology and representational content, contrary to popular intentionalist views of perception, or spatial subjectivism is true, where spatial subjectivism is the view that the spatial properties presented in perception are either minddependent or illusory.
\end{abstract}




\section{Introduction}

Imagine there is a planet far away that is an exact mirror image of Earth. We'll call it "Mirror Earth.” On Mirror Earth, people have hearts on their right sides, English text is read from right to left, screws tighten counterclockwise, baseball players round the bases clockwise, and most people write with their left hand and process language in their right cerebral hemisphere. Mirror Earth, of course, is home to a mirror-image counterpart of you, whom we'll call "Twin." The puzzle developed in this paper begins with a simple question: what are Twin's experiences like? Are her experiences the same as yours, or are they phenomenally mirrorinverted relative to yours?

It will be helpful to focus on a specific experience. Imagine you are staring directly at a blank black screen, and you then see a flash appear on the left side of the screen. Meanwhile, a flash occurs on the right side of Twin's screen. When Twin sees the flash on her screen, what is her experience like? I think many will be tempted to say that her experience differs from yours. Specifically, it is tempting to think that Twin's phenomenology is mirror-inverted relative to yours - that her experience is like the experience you would have had if the flash had been on your right. I shall argue that this is incorrect. Rather, we ought to accept:

SAME EXPERIENCE: Twin's experience is phenomenally the same as yours.

But as we'll see, this conclusion leads to a puzzle, one that I believe can push us toward some surprising and philosophically significant conclusions about the nature of perception. The puzzle developed in this paper takes the form of a paradox, a set of jointly inconsistent but individually plausible claims about the experience of left and right and the metaphysics of left and right. I shall make the case that any viable response to the paradox will endorse the following disjunction: either there is a degree of independence between perceptual 
phenomenology and representational content, contrary to popular intentionalist views of perception, or spatial subjectivism is true, where spatial subjectivism is the view that the spatial properties presented in perception are mind-dependent or illusory. I confess I am not entirely comfortable with this conclusion. I have defended both intentionalism and spatial objectivism in previous work, ${ }^{1}$ and I still believe there is a great deal to be said for both. But in light of the paradox below, I don't see any plausible way to avoid the conclusion that one of them is false. Others may take a different view about the upshot of the paradox. Even so, I hope to convince the reader that this paradox can help us think more clearly through some important and neglected questions in the philosophy of perception about the experience of left and right. ${ }^{2}$

\section{The Puzzle}

When you see the flash on your left, your experience has a certain distinctive phenomenal character, which we'll express by describing the experience as "left-flashy." "Left-flashy" is intended to capture the fully specific phenomenal character of your experience, so an experience qualifies as left-flashy if and only if it is phenomenally exactly like your current visual experience. I will argue in the following section that we have compelling reason to accept:

SAME EXPERIENCE: Twin's experience is phenomenally the same as yours.

\footnotetext{
${ }^{1}$ Cutter and Tye (2011), Cutter (2017, 2018).

${ }^{2}$ The puzzle addressed in this paper has a good deal of overlap with some puzzles recently discussed by Lee (2006), Chalmers (2019), and Simon (forthcoming) about the experience of left and right, but our main concerns are somewhat different. Chalmers is mainly concerned with the epistemic possibility of mirror-reversal skeptical scenarios (i.e., whether it's possible that everything that appears on your left is actually on your right, and vice versa). Lee is mainly concerned with the possibility of left/right-inverted qualia (i.e., whether there could be a subject functionally just like you but with mirror-inverted phenomenology), and whether the physical asymmetry of our brains can be transcendentally deduced from the asymmetric character of our experiences. Simon's main goal is to resist Lee's argument for such a transcendental deduction and to show how these puzzles bear on questions about the metaphysics and structure of consciousness. Each defends claims that are relevant to the concerns of this paper, some of which will be discussed below. But none is primarily addressed to the central concern of this paper, namely: how do these puzzles bear on more familiar issues in the philosophy of perception about the relationship between content and phenomenology and the nature of the sensible properties presented in perception?
} 
In other words, we have compelling reason to believe that Twin's experience is left-flashy. But this conclusion gives rise to a puzzle, for the following three claims are individually very plausible, but (together with our description of the case) jointly contradict SAME EXPERIENCE:

SYMMETRY: You and Twin don't differ with respect to whether your experiences are veridical.

VERIDICALITY: Your experience is veridical.

Phenomenology-Content Link: Necessarily, if one has a left-flashy experience, then one's experience is veridical only if there is a flash on one's left.

SYMMETRY is very plausible. Given the perfect physical symmetry of your situations, it would be bizarre if just one of you were experiencing veridically. VERIDICALITY is also very plausible. Since nothing abnormal is going on with your experience of the flash, it is safe to assume that if any of your left/right asymmetric visual experiences are veridical, then this one is. Thus, the denial of VERIDICALITY is tantamount to the claim that all your visual experiences of left and right are non-veridical. I assume on general anti-skeptical grounds that we should regard experience as veridical in the absence of strong countervailing considerations. Thus, I assume we have strong prima facie reason to avoid the kind of radical error theory about spatial perception that would come with the rejection of VERIDICALITY.

Phenomenology-Content Link is quite plausible as well. PhenOMENOLOGY-Content LINK says, in effect, that any experience phenomenally just like yours would necessarily present a flash as occurring on one's left—any such experience would necessarily be an experience "as of" a flash on one's left - and would therefore be veridical only if there is a flash on one's left. This claim is closely related to the attractive idea that the phenomenal character of a perceptual experience is intimately and inseparably connected with what it represents. Suppose you have a 
visual experience as of a yellow crescent in front of you. Your experience has a certain representational content, representing the perceived object as yellow, crescent-shaped, and in front of you. The connection between the phenomenal character of your experience and this representational content does not seem accidental. The perceived color, shape, and viewerrelative location seem to be in some sense "directly presented" in the phenomenology of the experience. It seems that an experience with this very phenomenal character must represent its object as yellow, crescent-shaped, and in front of the subject. It seems inconceivable that someone should have an experience phenomenally just like your yellow-crescent experience and thereby experience the perceived object to be, say, blue and cubical. Something similar plausibly holds of your experience as of a flash on your left. Your experience represents the presence of a flash on your left, and this representational content does not seem to be merely accidentally connected to the "left-flashy" phenomenal character of the experience. Rather, it seems that any experience with this very phenomenal character must represent the occurrence of a flash on the subject's left. At least on initial reflection, it seems inconceivable that someone should have an experience phenomenally just like your flash-on-the-left experience and thereby experience the perceived object to be on her right.

Some terminology will be useful. We'll say that a property or relation $F$ is presented by an experience $e$ (alternatively: figures in the presentational content of $e$ ) just in case, necessarily, any experience with the same phenomenal character as $e$ represents $F$ as instantiated.

PHENOMENOLOGY-CONTENT LINK is then roughly equivalent to the claim that being on the left is presented by, or belongs to the presentational content of, your experience. The idea that perceptual experience has presentational content is a consequence of intentionalism, or at least the version of intentionalism that will concern us here. For our purposes, we'll define 
intentionalism as the view that the phenomenal properties of an experience - those that characterize what it's like for the subject to undergo the experience-are identical to representational properties of the form representing (in an appropriate manner) such-and-such content. ${ }^{3}$ For the purposes of this paper, I shall assume the intentionalist conceives of the relevant contents as "Russellian," in the sense that they contain or directly involve sensible properties and relations, not just "modes of presentation" that pick out different properties/relations in different environmental contexts.

Of course, PhENOMENOLOGY-CONTENT LINK won't be entirely uncontroversial. Some say that representational content is independent of phenomenal character and therefore deny that perceptual experiences have presentational content in the sense just defined. Others may find a way to reject PHENOMENOLOGY-CONTENT LINK without giving up presentational content. We'll return to these issues in $\$ 4.4$. For now, I only claim that PHENOMENOLOGY-CONTENT LINK is prima facie plausible, and should be especially attractive to those who find intentionalism plausible.

If I succeed in establishing the truth (or at least the plausibility) of SAME EXPERIENCE, the four claims above - SAME ExPERIENCE, SyMmETRY, VERIDICALITY, and PHENOMENOLOGYCONTENT LINK — constitute a paradox, a set of individually plausible but jointly inconsistent propositions. After making the case for SAME EXPERIENCE in $\S 3$, I survey possible responses to the paradox in $\S 4$. There I shall argue that we should not respond to the paradox by rejecting SYMMETRY or SAME EXPERIENCE. Rather, the only viable responses to the paradox involve rejecting VeridicAlity or PHENOMENOLOGY-CONTENT LinK. This conclusion has important implications for the philosophy of perception, for as I'll argue, every promising strategy for

\footnotetext{
${ }^{3}$ Proponents of intentionalism (or something near enough) include Tye (1995), Dretske (1995), Lycan (1996), Horgan and Tienson (2002), Chalmers (2006), Pautz (2010), and Speaks (2015).
} 
rejecting VERIDICALITY or PHENOMENOLOGY-CONTENT LiNK leads to the disjunctive conclusion mentioned above: either intentionalism is false or spatial subjectivism is true.

\section{The Case for SAME EXPERIENCE}

Before developing the argument for SAME EXPERIENCE, we must distinguish two views about the metaphysics of left and right, which I shall call intrinsicalism and extrinsicalism. Intrinsicalism and extrinsicalism offer opposing views about what differentiates incongruent counterparts - pairs of objects, such as a right hand and a left hand, that are mirror images of one another and cannot be superimposed on one another via rigid motions (rotations and translations). According to intrinsicalism, incongruent counterparts differ intrinsically. A left hand is left rather than right in virtue of possessing a certain intrinsic shape property, the property of being left-hand shaped. According to extrinsicalism, incongruent counterparts do not differ intrinsically; they are duplicates, objects that are exactly qualitatively alike in intrinsic respects. They differ only in their relations to other material objects. Most obviously, they differ in their congruence and incongruence relations to other hands. Your right hand can be (roughly) superimposed on the hand opposite my heart, but your left hand cannot. There are other slightly less obvious relational differences. For example, your left hand will fit in my baseball glove but your right hand will not. And your right hand, if held up with its palm facing a standard clock, will find its thumb pointing to the "9," while the thumb of your left hand will point to the "3." According to the extrinsicalist, there are no further differences between a right and a left hand beyond extrinsic relational differences of this kind. ${ }^{4}$

\footnotetext{
${ }^{4}$ In this paper, the only form of extrinsicalism I consider is one according to which left hands and right hands are distinguished by their relations to other material objects (and - if you like-particular regions of space). There is another form of extrinsicalism, famously defended by Kant (1768/1991), according to which a hand is left or right not in virtue of its relations to other material objects or to particular regions of space, but in virtue of its relations to "universal space as a unity of which every extension is a part" (27). Kant rejects the version of extrinsicalism considered in this paper because it conflicts with the claim that a hand in an otherwise empty universe would have to be either a left hand or a right hand. This claim is nowadays generally thought to have been refuted by the facts
} 
From here, the argument for SAME EXPERIENCE relies on two premises:

(S1) Extrinsicalism is true.

(S2) If extrinsicalism is true, then SAME EXPERIENCE is true.

Why accept (S1)? First, extrinsicalism appears to be the dominant view within the philosophy of physics. Chalmers (2019) calls it the "consensus" view (p. 117), and Baker (2012) writes, "I think I speak for a large population of specialists in asserting that [extrinsicalism] is far more plausible than [intrinsicalism]" (p. 487). ${ }^{5}$ Perhaps we should not defer to philosophers of physics on all issues, but on a question like this, which concerns the nature of space, their opinion should carry significant weight. Second, we can derive the falsity of intrinsicalism from the plausible premise that the intrinsic spatial properties of an object supervene on the distances and angles among its parts. In other words, if there is a one-one mapping from the parts of $x$ onto the parts of $y$ that preserves distances and angles, as is the case when $x$ and $y$ are mirror images, then $x$ and $y$ are intrinsically identical (at least in spatial respects).

The third and most important reason to accept extrinsicalism stems from some well known facts about the geometry of higher-dimensional spaces and non-orientable spaces. First, consider a pair of incongruent counterparts in a two-dimensional space, such as the ' $b$ ' and the 'd' in figure 1:

\section{b d}

Figure 1: Incongruent counterparts in a two-dimensional space

\footnotetext{
about higher-dimensional and non-orientable spaces discussed below. In any event, as Earman (1991) observes, Kant's appeal to "universal space as a unity" doesn't seem to help explain handedness, for it is entirely unclear why a solitary right hand would have to differ from a solitary left hand in its relations to absolute space considered-as-aunity. Cf. (Gardner, 1964, 166-7) and (van Cleve, 1987, 49-50). For these reasons, I set aside Kantian extrinsicalism for the purposes of this paper.

${ }^{5}$ See also Hoefer (2000) and Pooley (2003). According to Baker, the main motivation for extrinsicalism from physics is that intrinsicalism would lead to violations of Leibniz Equivalence, the principle that symmetry transformations to a solution of a spacetime theory do not yield new physical possibilities.
} 
The "b" and "d" are "incongruent" in the sense that neither can be superimposed on the other via rigid motions, at least if those rigid motions are confined to the two-dimensional plane on which these figures live. However, if we could lift the "d" off the page and rotate it in three dimensions, it could be superimposed on the "b." Something similar goes for incongruent counterparts in three-dimensional space. Let Lefty and Righty be any pair of incongruent counterparts living in a three-dimensional space (e.g., a left and a right hand). No series of rigid motions confined to the three-dimensional space they inhabit will suffice to superimpose one on the other. But if Lefty and Righty (or objects intrinsically just like them) were embedded in a four-dimensional space, then they could be superimposed on one another via rigid motions within that higherdimensional space. Now, it's very plausible that rigid motions don't make a difference to how an object is intrinsically, and it's also very plausible that the dimensionality of space (e.g., the fact that a hand exists in a three-dimensional space rather than a four-dimensional space) is not intrinsic to an object. ${ }^{6}$ It is therefore hard to avoid the conclusion that Lefty and Righty are (in the actual world) intrinsically the same.

A bit more carefully, the argument for (S1) rests on two claims: (i) There is a possible four-dimensional space that contains objects_ call them Lefty* and Righty*_-that are duplicates of Lefty and Righty, respectively. (ii) Lefty* and Righty* are duplicates of each other (because they can be superimposed by rigid motions). Given the transitivity of duplication, it follows that Lefty and Righty are duplicates of each other. Since Lefty and Righty were an arbitrary pair of three-dimensional incongruent counterparts, the conclusion generalizes to all three-dimensional incongruent counterparts.

\footnotetext{
${ }^{6}$ It might be argued that properties of the form existing in a space of at least $n$ dimensions are intrinsic, perhaps on the grounds that this would explain why a duplicate of a three-dimensional object couldn't exist in a twodimensional space. Even if we grant this, however, the argument wouldn't extend to properties of the form existing in a space of exactly (or at most) $n$ dimensions, which are the properties relevant to my argument.
} 
An intrinsicalist might dig in and insist that, for whatever reason, Lefty and Righty (or duplicates of them) couldn't exist in a four-dimensional space. But this wouldn't save intrinsicalism, for the same point can be made with a non-orientable three-dimensional space. In a non-orientable space, an object can be superimposed on any of its counterparts by longdistance rigid motions. The most famous example of a non-orientable space is a Möbius strip, which can be constructed by taking a strip of paper, twisting one side 180 degrees, and joining the two ends together. If we have a "b" and a "d" side-by-side on a Möbius strip, we can bring them into congruence by sending the "d" on a long journey around the strip. Likewise, if we have a left hand and a right hand side-by-side in a three-dimensional non-orientable space, we can send one on a long journey such that, when it comes back, it will superimpose on the other hand. Importantly, a space like this can be locally indistinguishable from an ordinary Euclidean space. In other words, the local region inhabited by two hands side-by-side in a non-orientable space could perfectly match the local region inhabited by two hands in a three-dimensional Euclidean space in its intrinsic geometric structure. The fact that a space is orientable or nonorientable is a fact about its global structure, not something that can be read off the intrinsic geometry of a given local part of it. Thus, we can argue as follows for extrinsicalism without any forays into the fourth dimension: (i) There is a possible non-orientable space that contains objects, Lefty* and Righty*, that are intrinsic duplicates of Lefty and Righty, respectively. (ii) Lefty* and Righty* are intrinsic duplicates of each other (since they can be superimposed on one another via rigid motions). Since duplicates of duplicates are duplicates, it follows from (i) and (ii) that Lefty and Righty are intrinsic duplicates of each other.

Let's turn to (S2): if extrinsicalism is true, then SAME EXPERIENCE is true. This is a trivial consequence of the popular thesis of phenomenal internalism, which holds: 
NARROW SUPERVENIENCE: Necessarily, physical duplicates (i.e., individuals that are intrinsically the same in physical respects) are phenomenal duplicates.

It is also a consequence of standard forms of phenomenal externalism, according to which a subject's relations to external features of her environment can constitutively contribute to the phenomenal character of her experience. Standard forms of phenomenal externalism would endorse a somewhat weaker supervenience thesis:

WIDE SUPERVENIENCE: Necessarily, physical duplicates occupying corresponding positions in duplicate environments are phenomenal duplicates.

Since it follows from extrinsicalism that you and Twin are physical duplicates occupying corresponding positions in duplicate environments, (S2) must be accepted by anyone who endorses WIDE SUPERVENIENCE (and, a fortiori, by anyone who accepts NARROW SUPERVENIENCE). ${ }^{7}$

In fact, if we tweak the scenario slightly, we can derive (S2) from an even weaker supervenience thesis, namely:

WEAK SUPERVENIENCE: Necessarily, for any $x$ and $y$, if $x$ and $y$ are physically indiscernible - that is, exactly qualitatively alike in all (intrinsic and extrinsic) physical respects—-then $x$ and $y$ are phenomenal duplicates.

For we can imagine that our universe is globally mirror-symmetric, that trillions of light years away, beyond the furthest observable galaxies, the universe has a plane of symmetry, on the other side of which are mirror images of everything on this side, including a mirror image of Earth. If extrinsicalism is true, it's plausible that mirror-image counterparts in a mirrorsymmetric world are not just physical duplicates, but physically indiscernible. That is, they are

\footnotetext{
${ }^{7}$ Cf. (Lee, 2006, 295-6).
} 
exactly qualitatively alike not only intrinsically, but extrinsically as well. ${ }^{8}$ Of course, extrinsicalism trivially entails that mirror images would be intrinsically alike. And given extrinsicalism, it's hard to see how mirror image counterparts in a mirror-symmetric world could differ qualitatively in extrinsic respects. After all, they will exhibit the same pattern of qualitative relations to objects in their environment. If one of them bears a qualitative relation-for example, the next-to relation-to some object in its environment, then the other will bear the same relation to a duplicate of that object. ${ }^{9}$

Thus, even WEAK SUPERVENIENCE is strong enough to justify the inference from extrinsicalism to SAME EXPERIENCE. Weak Supervenience can be thought of as articulating a minimal physicalist view of consciousness. Of course, some philosophers reject physicalism about consciousness for independent reasons (Jackson 1982, Chalmers 1996), and one might think that rejecting physicalism would resolve our paradox by undermining the motivation for (S2), thereby undermining the argument for SAME EXPERIENCE. In that case, we'd have an interesting new argument for dualism. However, I'll argue below that the truth of dualism would not undermine the case for SAME EXPERIENCE.

\footnotetext{
${ }^{8}$ The indiscernibility of mirror-image counterparts in mirror-symmetric worlds is standardly taken for granted within discussions of the identity of indiscernibles. See, e.g., (Adams, 1979, 14) and (Lewis, 1983, 28).

${ }^{9}$ A bit more formally, we can derive the indiscernibility claim from extrinsicalism via some Lewisian principles concerning duplication and indiscernibility. Lewis (1986) says that $x$ and $y$ are duplicates iff there is a mapping from the parts of $x$ to the parts of $y$ that maps $x$ to $y$ and preserves all perfectly natural properties and relations (p. 61). To my knowledge, Lewis never offers a formal definition of indiscernibility, but Hawthorne and Dorr (2013) suggest the following as a Lewis-inspired definition: $x$ and $y$ are indiscernible just in case there is a permutation on the domain of all objects that maps $x$ to $y$ and preserves perfectly natural properties and relations (p. 11). Here we are interested in physical indiscernibility rather than indiscernibility simpliciter, but an account of physical indiscernibility is naturally achieved by replacing "all objects" with "all physical objects" and adding a restriction to perfectly natural physical properties and relations. An extrinsicalist who accepts the Lewisian account of intrinsicality will accept that a mapping from the parts of an object to the corresponding parts of its mirror image is a mapping that preserves perfectly natural properties and relations. Given our assumption that the universe is mirror symmetric, there is such a mapping — call it $g$ - from the (class of all parts of the) physical universe onto itself that maps you to Twin. Thus, there is a mapping - $g$ - from the set of all physical objects onto itself that preserves perfectly natural physical properties and relations and which maps you to Twin. Given our Lewis-inspired definition of physical indiscernibility, it follows that you and Twin are physically indiscernible.
} 


\section{Responses to the Paradox}

Our paradox consists of four mutually incompatible claims. One of them (at least) must be rejected. But which one, and why? I'll start by considering what I take to be the least promising response to the paradox, namely, rejecting SYMMETRY (§4.1). Next, I consider strategies for resisting the argument above for SAME EXPERIENCE (§4.2). After arguing that none of these strategies succeeds, I consider the options of rejecting VERIDICALITY (§4.3) and rejecting PhenOMENOLOGY-CONTENT LinK (§4.4). Although these options have significant costs and revisionary consequences, they are least more promising than the alternatives, or so I shall argue. I shall also argue that every viable strategy along these lines has the consequence that intentionalism is false or spatial subjectivism is true.

\subsection{Rejecting SYMMETRY}

According to SYMMETRY, you and Twin do not differ with respect to whether your experiences are veridical. I think it is clear that SYMMETRY must be true, at least if my argument in $\S 3$ for SAME EXPERIENCE is sound. There I noted that, given extrinsicalism, mirror-image counterparts in a mirror-symmetric universe would be physically indiscernible. If we tweak our scenario along the lines suggested above and imagine that our universe is globally mirrorsymmetric, we then get the result that you and Twin are physically indiscernible. Given SAME EXPERIENCE, you are also indiscernible in phenomenal respects. Now, if two individuals are physically and phenomenally indiscernible, then presumably they are indiscernible tout court. And it is about as self-evident as anything in philosophy that indiscernibles (that is, subjects who are exactly qualitatively alike in both intrinsic and extrinsic respects) cannot differ with respect to whether they experience veridically. We therefore should not deny SYMMETRY, at least not 
without challenging SAME EXPERIENCE or the argument given in its support (specifically, the premise of extrinsicalism). Let us then consider strategies for rejecting SAME EXPERIENCE.

\subsection{Rejecting SAME EXPERIENCE}

If we reject SAME EXPERIENCE, we must reject some step in the argument given in $\S 3$. Recall that this argument relied on two premises: (S1) Extrinsicalism is true, and (S2) If extrinsicalism is true, then SAME EXPERIENCE is true. In $§ 3$, I gave a few arguments for (S1), but the main argument relied on the following claim (here Lefty and Righty are an arbitrary pair of three-dimensional incongruent counterparts):

POSSIBLE-DuPLICATES: There is a possible four-dimensional space (variant: nonorientable three-dimensional space) that contains objects, Lefty* and Righty*, that are intrinsic duplicates of Lefty and Righty, respectively.

Given PossibLe-DuPLICATES, the truth of extrinsicalism follows fairly straightforwardly. For it's clear that Lefty* and Righty* would be duplicates of each other, given that they could be perfectly superimposed on one another via rigid motions - motions of a kind that very plausibly leave an object's intrinsic character unchanged. Since duplication is transitive, it follows that Lefty and Righty are duplicates.

Thus, it looks like the intrinsicalist will have to deny POSSIBLE-DuPLICATES. One way to do so would be to reject the prevailing wisdom that four-dimensional and non-orientable spaces are possible. ${ }^{10}$ But the prevailing wisdom prevails for good reason. There are formally consistent geometries of higher-dimensional and non-orientable spaces, and it's reasonable to take mathematical consistency as a defeasible guide to possibility. In this case, I think the presumption of possibility is undefeated. Apart from the assumption, discredited by modern

\footnotetext{
${ }^{10}$ Van Cleve (1987) flirts with this response.
} 
physics, that space must be flat, there seems no good reason to disallow non-orientable spaces. And while four-dimensional space is hard to visualize, this gives us no more reason to deny its possibility than flat-landers have to deny the possibility of three-dimensional space. (Moreover, even if higher-dimensional and non-orientable spaces are impossible, this would not affect my other argument for extrinsicalism, which relies on the intuition that the intrinsic spatial properties of an object supervene on the distances and angles among its parts.)

Josh Parsons (ms) challenges PossiBLe-DuPLICATION without denying the possibility of higher-dimensional and non-orientable spaces. According to Parsons, there are three determinates or "chiral variants" of the determinable property being hand-shaped: being lefthand shaped, being right-hand shaped, and being non-orientably hand-shaped. These are supposed to be distinct and mutually exclusive intrinsic properties. The intrinsicalist, says Parsons, must therefore deny that Lefty and Righty are duplicates of their counterparts in the non-orientable world. "The (apparently) left hand in [the non-orientable world] is hand-shaped, but not left-hand-shaped or right-hand-shaped. The same goes for the (apparently) right hand" (3).

This view faces serious difficulties. If being right-hand-shaped, being-left-hand-shaped, and being non-orientably-hand-shaped are intrinsic properties, then it should be possible for God to create a non-orientable hand next to a right hand and a left hand. We can then ask: will the non-orientable hand superimpose on the left hand or the right hand? Parsons offers two possible responses: either this scenario is impossible (non-orientable hands can't coexist with left hands or right hands) or the non-orientable hand will be superposable on both of the others, though it may have to take a long journey to superimpose on one of them. But the first option violates plausible Humean principles of modal recombination, and the second is hard to make sense of. 
Suppose the non-orientable hand is first superimposed on the right hand. The claim is that, if the non-orientable hand then takes a (perhaps long) journey along a certain trajectory, it could then be superimposed on the left hand. But now a problem arises if we let the non-orientable hand take that journey with the right hand tagging along for the ride, remaining directly upon the nonorientable hand the whole way. If at the end of the journey, the non-orientable hand superimposes on the left hand, then the right hand will as well. But then we should conclude that the left hand and the right hand are intrinsically the same after all.

Moreover, even if intrinsicalism is true, there is still some pressure to accept SAME EXPERIENCE. For even if you and Twin differ intrinsically, you arguably qualify as narrow functional duplicates. ${ }^{11}$ If so, then anyone who accepts the supervenience of phenomenology on narrow functional properties must accept SAME EXPERIENCE, whether or not extrinsicalism is true. $^{12}$

I conclude that rejecting extrinsicalism is not a promising strategy for resisting the argument for SAME EXPERIENCE. The alternative, for those who wish to reject SAME EXPERIENCE, is to reject (S2), which says that SAME EXPERIENCE is true if extrinsicalism is true. Recall that this conditional was supported by:

\footnotetext{
${ }^{11}$ Only "arguably," because the issue turns on the level of abstraction at which we characterize the relevant functional properties. Functional properties are defined in terms of causal relations to sensory inputs, behavioral outputs, and other internal states, where the intrinsic natures of the relevant internal states are left unspecified, but those of the inputs and outputs can be specified. So, the question of whether you and Twin are narrow functional duplicates will depend on whether the inputs and outputs are specified in a way that abstracts away from left/right orientation (e.g., retinal stimulation in an eye vs. retinal stimulation in the left eye, or lifting a hand vs. lifting a right hand). Many functionalists seem to tacitly assume that the inputs and outputs will specified at a very high level of abstraction. For example, many functionalists hold that a detailed computer simulation of our bodies and environments would suffice to duplicate our phenomenology (Bostrom, 2003, Schwitzgebel, 2019). But the "inputs" and "outputs" in such a computer simulation would be computational processes that could well be entirely insensitive to physical left/right orientation (e.g., operations on 1's and 0's on a vertical tape).

${ }^{12}$ Cf. (Lee, 2006, 309).
} 
WEAK SUPERVENIENCE: Necessarily, for any $x$ and $y$, if $x$ and $y$ are physically

indiscernible, then $x$ and $y$ are phenomenal duplicates.

As mentioned above, some philosophers-for example, dualists—reject Psychophysical

Supervenience on independent grounds. It might therefore be suggested that dualism can help us resolve the paradox by undermining the argument for SAME EXPERIENCE. ${ }^{13}$

One immediate objection to this response is that we can interpret "necessarily" in Weak Supervenience to express nomological necessity rather than metaphysical necessity, in which case even dualists should accept it. But this objection can be resisted. If we give Psychophysical Supervenience a nomological reading, we will need to assume that our imagined scenario is nomologically (not just metaphysically) possible. And there are empirical problems with this assumption, stemming from the fact that the laws of physics discriminate between (what are conventionally labeled) "left-handed" and "right-handed" particles, where the "handedness" of a particle is determined by the direction of its spin around a given axis. For example, the behavior of a right-handed beta-decaying Cobalt 60 atom is known not to be physically equivalent to that of its left-handed counterpart. ${ }^{14}$ The upshot is that, if we want our imagined scenario to be perfectly mirror symmetric down to the microscopic level, we'll need to say that something physically odd happens at plane of SYMMETRY of the universe, something like a parity-flip in the laws governing the two sides of the universe. If all we're after is metaphysical possibility, this isn't much to worry about. But if we're looking for a nomologically possible scenario, then we need to worry about these empirical complications.

\footnotetext{
${ }^{13} \mathrm{Cf}$. Simon (forthcoming), who expresses sympathy for a dualist solution to a related puzzle about mirror-image counterparts in non-orientable worlds.

${ }^{14}$ See Gardner (1964), Baker (2012), and Pooley (2003) for further discussion of left-right asymmetry in physics. Baker's discussion is especially valuable for its lucid explanation of why left-right asymmetrical laws do not upset the consensus view that extrinsicalism is true, contrary to what (Lee, 2006, 311n) suggests.
} 
So the strategy of responding to the paradox by rejecting Weak Supervenience cannot be dismissed so easily. Still, I think it's not a promising response to the paradox. Let's assume dualism is true, so that Weak Supervenience is false (with "necessarily" interpreted metaphysically). There are at least two dualist-friendly ways of reformulating the argument for SAME EXPERIENCE. First, we could define a kind of necessity that is intermediate in strength between nomological and metaphysical necessity, which we might call psychophysical necessity. Nomological necessity is commonly thought of as a restriction of metaphysical necessity to worlds that verify the actual laws of nature. Thus, where $L$ is the conjunction of all the laws of nature, it's nomologically necessary that $p$ iff the material conditional $L \supset p$ is metaphysically necessary. Similarly, if $L^{*}$ is the (smaller) conjunction of psychophysical laws, we can say that it's psychophysically necessary that $p$ iff $L^{*} \supset p$ is metaphysically necessary. Even if our imagined scenario violates physical law and is therefore nomologically impossible, it is plausibly still psychophysically possible. (Indeed, it's hard to see how it could violate any psychophysical laws given that our description of the scenario said nothing about what sorts of experiences Twin is having.) In that case, we can interpret the modal in Weak Supervenience as expressing psychophysical necessity, and the case for SAME EXPERIENCE will go through without reliance on physicalism.

Second, we can try to sidestep the empirical complications mentioned above by considering a scenario that is only approximately mirror symmetric. It's clearly nomologically possible to have complex physical systems that are very close to mirror symmetric (hands, for example). So it is probably nomologically possible to have two subjects who are approximate mirror images occupying corresponding positions in approximate-mirror-image environments. In that case, the argument for SAME EXPERIENCE will go through, so long as we make two plausible 
assumptions. The first is that (to borrow some terminology from (Yli-Vakkuri \& Hawthorne, $2018,89)$ the physical-phenomenal connection isn't fickle; that is, phenomenology is not sensitive to trivial microscopic differences of the sort by which you and your almost-mirrorimage would differ. Second, the trivial microscopic respects in which you and Twin differ aren't the sorts of things that would make a difference with respect to whether your experiences are veridical. Since both of these assumptions are plausible, dualism doesn't do much to vitiate the argument for SAME EXPERIENCE.

\subsection{Rejecting VERIDICALITY}

The next possible response to the paradox is to reject VERIDICALITY, the claim that your experience is veridical. Rejecting VERIDICALITY sounds like a radical option. On the face of it, to reject VERIDICALITY is to hold that nothing is ever on your left, and surely that's absurd. Echoing Moore, one wants to respond, "Here is a left hand.” Doesn't that settle the matter?

Rejecting VERIDICALITY is a radical option, but it needn't be quite as radical as it sounds at first blush. Here a helpful model is Chalmers's (2006) two-stage Edenic view of perception. According to Chalmers, perceptual experience has two kinds of content, "Edenic content" and “ordinary content.” In virtue of its Edenic content, a visual experience represents primitive sensible qualities (e.g., primitive colors and primitive spatial properties). Chalmers holds that the qualities that figure in Edenic content aren't instantiated in our world, but according to his fable, they were instantiated in the Garden of Eden, before we ate of the Tree of Illusion and the Tree of Science. By contrast, the ordinary content of experience attributes ordinary physical properties to objects in our environment — whatever physical properties serve as the normal causal basis for experiences of the relevant type. For example, in virtue of its ordinary content, our phenomenally reddish experiences represent whatever property normally causes reddish experiences in us, 
perhaps a surface reflectance type or a complex microstructural property. We can call this property "physical redness" (as distinct from "Edenic redness," the primitive quality that figures in the Edenic content of such an experience). Edenic content is supposed to be intimately connected with phenomenology in a way that ordinary content is not. In the terminology introduced in $\S 2$, Edenic content is presentational content-representational content that is necessitated by phenomenal character. Ordinary content is not presentational, because a given type of experiences might have different normal causes for different subjects.

We might apply this model to experiences of left and right. We could say that the Edenic content of your experience is that a flash is to your "Edenic left," or "lefte," where lefte is a primitive left relation that is not instantiated in our world, but might have been instantiated in the Garden of Eden. ${ }^{15}$ We would thereby deny VERIDICALITY with respect to the Edenic content of your experience. But we could still say that your experience is veridical in virtue of its ordinary content, which represents a flash as occurring on your "physical left," or "leftp," the relation that serves as the normal causal basis of your phenomenally leftish experiences. One might add that ordinary uses of "left" pick out left , so that ordinary claims to the effect that so-and-so is to the left come out true. (Chalmers makes an analogous claim about ordinary color talk.)

One concern with the Edenic view of left-right experience is that it requires us to believe that there exist uninstantiated properties and relations like Edenic left and being Edenically lefthand shaped, and that we somehow bear intentional relations to these properties and relations. Of course, the same point applies to the Edenic view of color, so those who already accept such a view will probably not be moved by this concern. Although this is certainly an important

\footnotetext{
${ }^{15}$ Somewhat surprisingly, Chalmers himself does not accept this picture of left/right experience, despite his endorsement of an analogous view for some other perceived spatial properties. Chalmers views about left/right experience will be discussed in $\$ 4.4 .2$.
} 
challenge to Edenic views generally, I don't think it constitutes anything like a decisive objection. Granted, we don't have a good understanding of how we could sensorily represent uninstantiated properties like Edenic red or Edenic left, but this may just be a consequence of the fact that we don't have a very mature understanding of how mental representation works in general. The problem of intentionality is hard, and we are far from a full solution. Moreover, whatever one's views about sensory content, we evidently attribute uninstantiated properties to things in belief, as when one believes that the creature over yonder is a unicorn or that an object is at absolute rest. Although there are differences between doxastic and sensory representation, it is not obvious why the sensory representation of uninstantiated properties should be regarded as more metaphysically problematic than the doxastic representation of uninstantiated properties. ${ }^{16}$

If we accept the Edenic view, it is natural to say that you and Twin have phenomenally identical experiences, experiences that are therefore alike in Edenic content. But they will differ in ordinary content: your experience represents a flash on the left $\mathrm{p}_{\mathrm{p}}$, while Twin's represents a

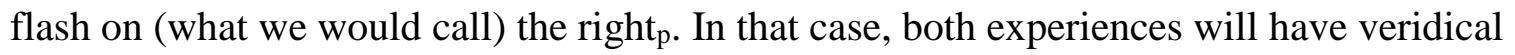
ordinary contents and non-veridical Edenic contents.

Still, there is a residual puzzle. Consider the version of our scenario involving a globally mirror-symmetric universe, so that you and Twin are fully indiscernible. One might have thought that indiscernible subjects wouldn't differ with respect to the ordinary contents of their experiences either. There is a related puzzle about linguistic content. Ordinary talk of "left" and "right" arguably corresponds to the ordinary content of perception, not the Edenic content. If so,

\footnotetext{
${ }^{16}$ As a relevant disanalogy, some might suggest that doxastic representations of uninstantiated properties are always built up from simpler concepts of instantiated properties (e.g., the concept unicorn might be built up from concepts like horse and horn). But there are several familiar problems with this model. For example, Kripke's (1980) arguments against descriptivism for natural-kind concepts like tiger apply equally to unicorn. Normative concepts raise a further difficulty. Concepts like good, bad, and obligatory probably aren't analyzable in terms of descriptive concepts, but surely this fact alone doesn't show that Mackie-style error theory is false.
} 
then the proposal above entails that when you say "there is a flash on my left," your utterance is true iff there is a flash on your left , but when Twin says "there is a flash on my left," her utterance is true iff there is a flash on her right $\mathrm{p}$. But one might have thought that physically and phenomenally indiscernible subjects wouldn't differ in their speech contents.

The solution to this puzzle lies in the observation that the ordinary contents may differ only hacceitistically. There is a difference between being on the left and being on the right, or between being a left hand and a right hand, but (given extrinsicalism) in a mirror-symmetric universe these distinctions can only be drawn hacceitistically. For example, a left hand is a left hand in virtue of standing in various spatial relations to a complex network of particulars (congruence relations to paradigm left hands, incongruence relations to paradigm right hands, fitting relations to paradigm left gloves, and so on). By contrast, it is not plausible that Edenic contents can differ only hacceitistically; mere hacceitistic differences seem insufficient for a phenomenal difference, so if Edenic contents could differ merely hacceitistically, this would undermine their claim to be presentational content. This solution applies equally to the puzzle about linguistic content. The fact that you and Twin express different properties and relations with words like "left" and "left hand" is no more puzzling than the fact that you and Twin attribute different properties with the predicate "Australian." In your mouth, "Australian" expresses one hacceitistic property: the property of being from Australia. In Twin's mouth, it expresses a corresponding but distinct hacceitistic property: the property of being from Twin Australia.

If we respond to the paradox by rejecting VERIDICALITY, this gives us a form of spatial subjectivism. Specifically, we get eliminativist spatial subjectivism, the view that (at least some of) the spatial properties presented in perception are not instantiated in our world. (The other 
form of spatial subjectivism is response-dependent spatial-subjectivism, according to which spatial properties are response-dependent properties. The latter view will be explored in §4.4.) I cannot see any decisive objections to this way of responding to the paradox, and (for what it's worth) my own very tentative view is that this is probably the best response. Still, we should not understate how revisionary it is. Common sense supposes that the sensible properties presented in visual experience are really "out there," and perhaps even that these properties are mindindependent. Since the $17^{\text {th }}$ century, many philosophers have gotten used to the idea that this naïve objectivist view might be false for color and other traditional secondary qualities, but there has been much greater reluctance (evidenced, for example, in traditional ways of delineating the primary/secondary quality distinction) to abandon a naïve objectivist view about the spatial properties presented in perception. Giving up naïve objectivism about spatial perception also comes with a more radical metaphysical alienation from the external world. If our rejection of naïve objectivism is limited to color and other traditional secondary qualities, we can at least retain a sense of real contact with "things as they are in themselves" through our sensory access to their spatial properties. But if we take the next step and give up naïve objectivism about spatial properties, we seem to lose all contact with things in themselves (Schwitzgebel, 2019). Our experience becomes less like a window on the world (or even a color-tinted window on the world), more like a virtual-reality headset. ${ }^{17}$ Our knowledge of things comes to be a step removed, and we lose a valuable form of knowledge we thought we had, a form of knowledge wherein the real properties of objects become phenomenally present for us. ${ }^{18}$

\subsection{Rejecting Phenomenology-Content Link}

\footnotetext{
${ }^{17} \mathrm{I}$ borrow these metaphors from Pautz (ms).

${ }^{18}$ Cf. Johnston (1996).
} 
The final option is to reject PHENOMENOLOGY-ConTENT LiNK: necessarily, if one has a left-flashy experience, then one's experience is veridical only if there is a flash on one's left. Recall that one motivation for PHENOMENOLOGY-CONTENT LiNK comes from intentionalism, the view that phenomenal properties consist in intentional relations to contents. This section examines several strategies for resisting PHENOMENOLOGY-CONTENT LINK. I divide these strategies into two groups: those that reject intentionalism altogether, and those that propose versions of intentionalism compatible with the denial of PHENOMENOLOGY-CONTENT LiNK. Let us consider these in turn.

\subsubsection{The Anti-Intentionalist Strategy}

Let's start with the anti-intentionalist strategy. The guiding idea is that there is in general no necessary connection between the representational content of an experience and its phenomenal character, so we should not expect a left-flashy phenomenal character to entail the representation of a flash on one's left. In the right circumstances, an experience with this phenomenal character could, according to this proposal, represent the occurrence of a flash on one's right. A natural way to develop this proposal would be to say that phenomenal character is determined by one's intrinsic neural or functional properties, whereas representational content is determined by one's extrinsic properties, for example, by tracking relations between one's phenomenal states and external properties in one's environment. On this view, your left-flashy experience represents that there is a flash to the left (because experiences of this kind are normally caused in you by flashes to the left), but Twin's left-flashy experience represents that there is a flash on her right (because experiences of this kind are normally caused in her by flashes to the right). ${ }^{19}$

\footnotetext{
${ }^{19}$ Chalmers (2019) calls this view "left-right functionalism." It is one component of his broader "spatiotemporal functionalist" view, which applies a similar model to all forms of spatiotemporal experience.
} 
I find intentionalism plausible, and have defended it in previous work, so I am disinclined to take this route. Still, this option is worth exploring, partly because others may not share my enthusiasm for intentionalism, and partly because those sympathetic to intentionalism should at least consider abandoning intentionalism if — like me - they don't find any of the other responses to the paradox especially attractive.

If we treat our paradox as an argument against intentionalism, it has notable connections with another important argument against intentionalism: Ned Block’s (1990) Inverted Earth argument. It is also notable that the argument implicit in our paradox is (in certain respects, anyway) a better argument against intentionalism, since it is not vulnerable to some of the standard responses to Block's argument. Block's Inverted Earth argument be understood as challenging a corresponding phenomenology-content link for color experience:

Chromatic Phenomenology-Content Link: Necessarily, if one has a greenish experience (i.e., an experience of the sort normally caused in us by limes, cucumbers, and clovers), then one's experience is veridical only if there is a green thing before one. Block asks us to imagine that you are kidnapped one night in your sleep and taken to Inverted Earth, a planet just like ours except everything's color is inverted: grass is red, the sky is yellow, fire engines are green, and so forth. Immediately upon arrival, you are fitted with color-inverting lenses, so the color experiences you undergo are the same as on Earth. You don't notice anything amiss. At first, Block thinks that your color experiences will be illusory. For example, when you look at the grass on Inverted Earth, your greenish experience will incorrectly represent the grass as green. But Block supposes that, after you've spent enough time in your new environment, there will be a content shift—your greenish experiences will represent red, for examplealthough there will be no corresponding shift in phenomenology. You will continue to have 
phenomenally greenish experiences in response to the grass, but these greenish experiences will now correctly represent the grass as red. We thus have a violation of CHROMATIC PHENOMENOLOGY-CONTENT LINK.

These two assumptions - the content-shift assumption and the phenomenal-constancy assumption - are essential to the argument. Why does Block think they are true? The motivation for the content-shift assumption seems to be a background commitment to something like a tracking theory of perceptual intentionality, according to which (very roughly) greenish experiences represent whatever property serves as their normal causal basis. The motivation for the phenomenal-constancy assumption seems to be that grass produces the name internal brain state throughout the whole process. The background assumption here seems to be something like the phenomenal internalist thesis that phenomenal properties supervene on narrow physical properties. Both of these claims have some prima facie plausibility, but neither is obviously true, and each is rejected by many philosophers. Some intentionalists reject phenomenal internalism (typically because they accept something like the tracking theory of perceptual intentionality) (Dretske 1995, Lycan 1996, Byrne and Tye 2006). Other intentionalists reject the tracking theory (often because they accept phenomenal internalism) (Horgan and Tienson 2002, Chalmers 2006, Pautz 2013). By contrast, if we use the puzzle presented in this paper as an argument against intentionalism, we don't need to rely on anything as controversial as these claims. Instead of phenomenal internalism, we only need the much weaker thesis of Weak Supervenience, which would be accepted even by phenomenal externalists. Similarly, we don't need to presuppose anything so strong as the tracking theory of perceptual intentionality, or any specific theory of perceptual intentionality. All we need is the modest assumption that our actual experiences of 
things to the left are veridical (VERIDICALITY) and that subjects who are physically and

phenomenally indiscernible are alike with respect to having veridical experiences.

\subsubsection{Intentionalist-Friendly Strategies}

Let us turn our attention to intentionalist-friendly strategies for rejecting

PhenOMENOLOGY-CONTENT LinK. Here I'll consider three such strategies: the neutral

phenomenology strategy, the relationalist strategy, and the appearance property strategy.

Ultimately, I'll argue that only the last, which entails a version of spatial subjectivism, holds any

promise. $^{20}$

Let's begin with the neutral-phenomenology strategy, which appears to be a consequence

of Chalmers's (2019) proposal and is sympathetically discussed by Simon (forthcoming).

Compare your experience of the flash on your left to the experience you would have had if the

flash had occurred on your right. While these experiences may be linked with different

behavioral dispositions and different memory associations, the neutral-phenomenology view says

that there is no phenomenal difference between these two experiences. What it's like for you to

visually experience the flash on your left is exactly the same as what it would have been like to

visually experience a flash on your right. ${ }^{21}$ The presentational content of your experience of the

\footnotetext{
${ }^{20}$ One intentionalist-friendly option that I won't consider is so-called "Fregean intentionalism." This is the view that the phenomenal content of perceptual experience is Fregean, built up from Fregean senses or modes of presentation that pick out different properties in different environmental contexts. Specifically, they pick out whatever property serves as the normal causal basis of the corresponding type of experience in the subject's context (Chalmers 2004, Thompson 2009, Schellenberg 2011). The reason I won't discuss this view is that, despite branding itself as a form of intentionalism, it is nearly indistinguishable from the anti-intentionalist view just described, inasmuch as both hold that the phenomenal character of an experience can vary independently of the properties it represents and that the latter are fixed by tracking facts. So my discussion of intentionalist-friendly responses to the argument will focus on Russellian intentionalist views, those that hold that phenomenal content involves properties and relations rather than modes of presentation thereof.

${ }^{21}$ Chalmers's (2019) discussion strongly suggests a view along these lines, though some of his remarks might be taken to suggest a version of the "relationalist" response discussed below. He expresses his view by saying that there is no "categorical" difference between experiences of left and right and that "any apparent qualitative differences between them aris[es] from differences in associations, memories, dispositions, and the like" (117). He compares his view of the relationship between experiences of left and right to the extrinsicalist's (in his terminology, the "prelationist's") view of the relationship between left and right hands, a relationship of intrinsic qualitative sameness,
} 
flash on your left, then, couldn't be different from that of your counterfactual experience of the flash on your right. Both would simply represent that a flash occurs on some side or other. Thus, it is false that any experience phenomenally just like yours is veridical only if there is a flash on the subject's left.

This view has some counterintuitive consequences. Take your experience of the flash, which you believe to be on your left, and now consider the "skeptical hypothesis," that this belief is false: on this particular occasion, there is not a flash on your left, but rather a flash on your right. The neutral-phenomenology view evidently entails that, although your belief may be false, your experience is veridical. That seems like the wrong verdict. The belief in question seems to be one that is directly tied to the testimony of your visual experience, such that the belief couldn't be false if the experience accurately represents the scene before you.

Counterintuitive consequences aside, the main reason I reject the neutral-phenomenology strategy is that I find its phenomenological thesis incredible. It just seems introspectively obvious that, in addition to whatever behavioral and memory differences distinguish experiences of left and right, there is a categorical phenomenal difference between a visual experience of something to my left and a visual experience of something to my right (cf. Lee 2006: 314). Chalmers calls this view "E-categoricalism." It is difficult to give arguments for purely phenomenological judgments of this kind, just as it would be difficult to give an argument for the claim that there is a phenomenal difference between the experience of red and green, not just a difference in

with the only difference between them being extrinsic and relational. He goes on to clarify his view by noting that it implies the following position: for any left-right asymmetric total experience, a putative mirror-reversal of that experience would be phenomenally identical to the first experience (118). The restriction to "total" experiences allows him to accommodate, for example, the phenomenal difference between certain temporally extended experiences, such as (i) a flash on the left quickly followed by a flash on the right, vs. (ii) a flash on the left quickly followed by a flash on the left. But if we consider two possible experiences, a flash on the left vs. a flash on the right, and we stipulate that the subject closes her eyes for a while immediately before and after the appearance of the flash (so that neither experience falls within a longer succession of left-right asymmetric experiences), Chalmers' view seems to be that these experiences would be phenomenally the same (130-5). 
memory associations and behavioral dispositions. But for what it's worth, I can say that a clear majority of philosophers of mind I have spoken to agree with me on this point. I hope the reader does as well.

A second intentionalist-friendly strategy for resisting PHENOMENOLOGY-CONTENT LiNK is the relationalist strategy, which relies on what we'll call "relationalist intentionalism." According to relationalist intentionalism, the difference between the experience of left and right consists in a difference in which spatial and (perhaps) affordance relations are represented as holding between the object and other perceived objects, or between the object and one's body. By an "affordance relation," I mean a relation between an object and a subject (or a part of her body) that specifies what the subject can do with the object or how the object may affect the subject.

To understand this view, let us first examine an analogous puzzle involving the experience of size. Consider some ordinary visual experience of a foot-long stick, and let "Q" pick out its fully specific phenomenal character. Those who believe that absolute length properties like being one-foot long are phenomenally presented should accept the following principle:

Length Phenomenology-Content Link: Necessarily, if one has a Q experience, then one's experience is veridical only if there is a (roughly) foot-long object before one. But this principle runs into trouble if we imagine a scaled up counterpart of you on Big Earth, a planet just like Earth except everything is twice as big (cf. Prosser 2011, Thompson 2010, Chalmers 2019). It's plausible that your enlarged counterpart has an experience that is phenomenally just like yours (i.e., a Q experience), and it's plausible that your twin's experience 
is veridical if and only if yours is. If we want to maintain that size experience is generally veridical, we must therefore reject Length Phenomenology-Content Link.

My own preferred response to this puzzle is to say that only relative distances/lengths are phenomenally presented in visual experience. ${ }^{22}$ On this view, the presentational content of your stick experience tells you how big the stick is relative to other objects in your environment, or relative to your body, but it does not specify the stick's intrinsic size (if there is such a thing).

One might suggest similarly that left/right phenomenology can be fully captured in terms of the representation of certain spatial and (perhaps) affordance relations. More specifically, one might suggest that the phenomenal difference between an experience of something to the left and an experience of something to the right can be fully captured by differences in experienced relations to asymmetric aspects of the perceived scene and asymmetric aspects of one's body. I'll focus on bodily asymmetries, since it seems clear that there is a phenomenal difference between seeing a flash to the left and seeing a flash to the right even when there are no asymmetries in the rest of the perceived scene (as in our case, where the flash is viewed against a uniform black background). Let's say you wear a wedding ring on your left hand and write with your right hand. Then an experience of an object on the left might be represented as close(r) to your wedding-ring hand and far(ther) from your writing hand, and vice versa for objects presented on the right. In that case, it would be false that any possible experience phenomenally just like yours is veridical only if a flash occurs on the subject's left. For a subject like Twin, who wears a wedding ring on her right hand and writes with her left, a flash on the right would suffice for veridicality. Asymmetries in motor abilities may infect presentational content as well. Perhaps

\footnotetext{
${ }^{22}$ Chalmers (2019) defends this view for Edenic length (but not physical length). Prosser (2011) rejects it in favor of the (less plausible, to my mind) view that the presentational content of our length experience consists entirely of affordance properties. Both views are relevantly analogous to the view of chiral experience under consideration.
} 
you'll experience a tennis racket as to-be-grabbed if seen on your right but not on your left. The present suggestion is that, when you take away all the experienced relations to asymmetric aspects of our bodies and environments, nothing left remains of the phenomenal distinction between left and right.

I don't find this response to the paradox at all plausible. I'm willing to grant that in some cases, relational content may partly explain the phenomenal difference between experiences of left and right. This is especially plausible when there are salient asymmetries in the subject's bodily orientation (e.g., one foot forward, one foot back) or when the perceived item is "actionable," (e.g., a tennis racket or a hammer presented near your good hand vs. your bad hand). But this can't be the full story. Relational content of this kind seems, in particular, entirely unsuited to account for the phenomenal difference between (a) your actual experience of the flash to your left, and (b) the experience you would have had if the flash had occurred on the right. We can assume you're holding your body as symmetrically as possible, so that the two experiences wouldn't differ in the relations they represent to hold between the flash and asymmetric aspects of your body (e.g., close to your front foot). Of course, your body is never perfectly symmetrical. It has significant internal asymmetries (e.g., a heart on the left, not the right) and minor surface asymmetries (e.g., a few more freckles on your right arm than your left). But it is hard to believe that these internal asymmetries and minor surface asymmetries are always reflected in phenomenology when you have left/right asymmetric experiences. Even if there is some asymmetric internal structure (say, a corkscrew in the brain) that is present whenever you have an experience as of something on the left, it is not plausible that your experience represents a relationship between the object and this internal structure. After all, it's reasonable to suppose that when one consciously represents a relationship between two things, 
the relata will be in some way "present to consciousness," at least as objects of peripheral awareness within background experience. For example, if one's experience represents an object as close to one's hand, then one will be at least peripherally aware of one's hand and its relation to the object. But it is phenomenologically implausible to suppose that we are even peripherally aware of the relevant internal structure whenever we have an experience as of something on the left. (To be clear, I am not denying that internal asymmetries are required in order to have phenomenally leftish experiences. I am only denying that such experiences represent relationships between external objects and internal asymmetries.)

Moreover, for a non-actionable item like a flash, it's equally implausible to suppose that experiences (a) and (b) would represent different affordances. (More cautiously: it's not plausible that the two experiences represent distinct affordances that can be specified in orientation-neutral terms. I suppose one might say that experience (a) is distinguished from experience (b) in virtue of representing the flash to have an affordance like: being such that one must turn left to view it straight on (cf. Evans 1985: 382-3). But if this is how we account for the phenomenal difference between left and right, the paradox remains. We need only rephrase PHENOMENOLOGY-CONTENT LINK with this affordance property in place of the property of being on one's left.) An intentionalist should look elsewhere for a resolution to the paradox.

In my view, the most promising intentionalist-friendly strategy for resisting PHENOMENOLOGY-CONTENT LINK is to adopt what I'll call appearance-property intentionalism, a view inspired by the intentionalism of Sydney Shoemaker $(1994,2001) .{ }^{23}$ First, consider color. According to Shoemaker, our phenomenally greenish experiences represent green, which he takes to be a physical property of external objects, but green doesn't figure in the presentational

\footnotetext{
${ }^{23}$ Views closely related to Shoemaker's are defended by Prinz (2006), Kriegel (2009), and Mehta (2012).
} 
content of greenish experiences. In other words, greenish experiences only contingently represent green. To this extent, he agrees with the anti-intentionalist view described in $\$ 4.4 .1$. However, Shoemaker does claim that greenish experiences necessarily represent a certain "colorlike" property (a "phenomenal color" as he sometimes puts it), which he calls an appearance property. Shoemaker identifies appearance properties with certain response-dependent properties of external objects. The appearance property presented by a greenish experience, for example, is something like the disposition to produce greenish experiences in the subject under certain conditions.

Shoemaker's view can be seen as a variation on Chalmers's two-stage Edenic view of color perception. Both draw a distinction between "physical color" and "phenomenal color," where only the latter figures in the presentational content of color experience. They differ in that Chalmers takes phenomenal colors to be primitive uninstantiated properties, whereas Shoemaker takes them to be response-dependent properties of external objects. But they agree in rejecting objectivist views of phenomenal color. In other words, they agree, against response-independent intentionalists like Tye (1995), Dretske (1995), Lycan (1996), and Byrne and Hilbert (2003), that phenomenal colors are not objective properties of external objects.

So, while accepting a form of intentionalism, Shoemaker would reject the analogue of PHENOMENOLOGY-CONTENT LINK for color:

Chromatic Phenomenology-Content Link: Necessarily, if one has a greenish experience, one's experience is veridical only if there is a green (i.e., physically green) thing before one.

But he would accept: 
Chromatic Phenomenology-ApPearance-Property Link: Necessarily, if one has a greenish experience, one's experience is veridical only if there is a phenomenal green thing before one (where phenomenal green is an appearance property).

In a similar way, we could distinguish between the physical left relation and phenomenal left relation, where the latter is a Shoemakerian appearance property associated with experiences of things to the left. For something to be phenomenal-left-related to $S$ is for it to be disposed to produce experiences with a leftish phenomenology in $S$. As with physical color, our experiences may represent, but do not phenomenally present, the physical left relation. Only the phenomenal left relation is presented in phenomenology. If we take this view, we will reject PHENOMENOLOGY-CONTENT LINK in favor of something like the following:

Phenomenology-ApPearance-Property Link: Necessarily, if one has a left-flashy experience, one's experience is veridical only if there is a flash that is phenomenal-leftrelated to one.

If we reject PhenOMENOLOGY-CONTENT LinK in favor of PHENOMENOLOGY-APPEARANCEPROPERTY LINK, our paradox is dissolved. Assuming the truth of the other propositions in the paradox, we get the result that you and Twin have phenomenally identical experiences, both of which are veridical. For, on this view, both experiences represent the flash as being phenomenalleft-related to the subject, and both flashes are phenomenal-left-related to the subject (that is, both flashes are disposed to cause phenomenally leftish experiences in the subject).

I take this option seriously. There is a lot to be said for appearance-property intentionalism about color experience. Unlike other forms of intentionalism, it avoids radical error theory about phenomenal color (an advantage over the Edenic intentionalism of Chalmers (2006) and Pautz (2011)) while upholding the plausible thesis of phenomenal internalism (an 
advantage over the response-independent intentionalism of Tye, Dretske, Lycan, and Byrne). Insofar as a unified treatment of color experience and spatial experience is desirable, appearanceproperty intentionalism about spatial experience deserves to be taken seriously as well. And, of course, it has the added advantage that it provides a resolution to our paradox.

Despite its virtues, the appearance-property view faces some challenges. One objection to the view is that it has difficulty accommodating certain plausible entailments from experienced orientational properties to mind-independent spatial facts. For example, if $x$ is phenomenally lefthand shaped and $y$ is phenomenally right-hand shaped (that is, if $x$ and $y$ have, respectively, the properties directly presented in typical experiences of left hands and right hands), this would seem to entail that $x$ and $y$ are (objectively, physically) opposite-handed. But if experienced orientational properties are just dispositions to produce experiences of a certain kind, it's hard to see why this entailment should hold. Perhaps the appearance-property intentionalist should reject these apparent entailments (maybe by distinguishing between physical opposite-handedness and phenomenal opposite-handedness, and insisting that the entailment only fails for the former), but the need to do so should probably be regarded as a cost for the view. Perhaps it is not an altogether new cost for those who already accept a response-dependent view of phenomenal color, however, for the latter view is arguably committed to the rejection of similar apparent entailments from phenomenal color to mind-independent spatial facts. For example, many have the intuition that the colors (or color-like properties) directly presented in color experience entail spatial extension. But as I have argued elsewhere, if these properties are just dispositions to produce experiences of a certain kind, it's hard to see why this entailment should hold. ${ }^{24}$ (Couldn't an unextended object have such a disposition?)

\footnotetext{
${ }^{24}$ Cutter (2016).
} 
Like the strategy of rejecting VERIDICALITY, the strategy under consideration-rejecting PhenOMENOLOGY-CONTENT LinK in favor of PHENOMENOLOGY-APPEARANCE-PROPERTY LINK - entails a subjectivist view of the spatial properties phenomenally presented in perception (though it is consistent with an objectivist view about physical spatial properties). Specifically, the current strategy entails response-dependent spatial subjectivism, according to which the spatial properties presented in perception are response-dependent. In other words, they are properties whose instantiation constitutively depends on the subjective responses of perceivers. This further supports the conclusion that, unless we are to abandon intentionalism, the correct resolution to the paradox will embrace some form of spatial subjectivism.

\section{Conclusion}

We've covered a lot of ground. Let's take a step back and tie things together with a summary of our main results. Our paradox consisted of four mutually inconsistent claims: SAME EXPERIENCE, SyMmetry, VeridiCALITY, and PHENOMENOLOGY-CONTENT LinK. I have argued that the only viable strategies for resolving the paradox involve rejecting PHENOMENOLOGYCONTENT LinK or Veridicality. If we reject Phenomenology-Content LinK, we should either reject intentionalism altogether or accept an appearance-property intentionalism about left/right experience, which entails a response-dependent form of spatial subjectivism. If we reject VERIDICALITY, we get an eliminativist form of spatial subjectivism. In either case, we are left with a surprising and significant disjunctive conclusion: either intentionalism is false or spatial subjectivism is true..$^{25}$

\section{References}

\footnotetext{
${ }^{25}$ For comments and helpful discussion, thanks are owed to Neil Mehta, Adam Pautz, Jeff Speaks, David Chalmers, Brad Saad, Alexander Pruss, Peter Epstein, Jonathan Simon, Daniel Nolan, Trevor Teitel, Nic Teh, E.J. Green, David Bourget, and Miriam Schoenfield.
} 
Baker, D. (2012) “The experience of left and right” meets the physics of left and right. Noûs, 46, 483-498. doi: 10.1111/j.1468-0068.2010.00814.x

Block, N. (1990). Inverted Earth. Philosophical Perspectives, 4, 53-79. doi: 10.2307/2214187

Bostrom, N. (2003). Are we living in a computer simulation? Philosophical Quarterly, 53, 243-255. doi: 10.1111/1467-9213.00309

Byrne, A. (2001). Intentionalism defended. Philosophical Review, 110, 199-240. doi: $10.2307 / 2693675$

Byrne, A. \& Hilbert, D. (2003). Color realism and color science. Behavioral and Brain Sciences, 26, 3-21. doi: 10.1017/s0140525x03000013

Byrne, A. and Tye, M. (2006). Qualia ain't in the head. Noûs 40, 241-255. doi: 10.1111/j.00294624.2006.00608.x

Chalmers, D. (1996). The Conscious Mind: In Search of a Fundamental Theory. Oxford: Oxford University Press.

Chalmers, D. (2004). The representational character of experience. In B. Leiter (Eds.), The Future for Philosophy (pp. 158-81). Oxford: Oxford University Press.

Chalmers, D. (2006). Perception and the fall from eden. In T. S. Gendler \& J. Hawthorne (Eds.), Perceptual experience (pp. 49-125). Oxford: Oxford University Press.

Chalmers, D. (2019). Three puzzles about spatial experience In A. Pautz \& D. Stoljar (Eds.) Blockheads! Essays on Ned Block's Philosophy of Mind and Consciousness (pp. 109-138). Cambridge, MA: MIT Press.

Cutter, B. (2016) Color and shape: a plea for equal treatment. Philosophers' Imprint 16:1-11.

Cutter, B. (2017) Spatial experience and special relativity. Philosophical Studies 174 (9): 2297-2313. doi: 10.1007/s11098-016-0799-8 
Cutter, B. (2018). Paradise regained: a non-reductive realist account of the sensible qualities. Australasian Journal of Philosophy 96 (1): 38-52. doi: $10.1080 / 00048402.2017 .1298141$

Cutter, B. \& Tye, M. (2011). Tracking representationalism and the painfulness of pain. Philosophical Issues, 21, 90-109. doi: 10.1111/j.1533-6077.2011.00199.x

Dorr C. \& Hawthorne, J. (2013). Naturalness. In K. Bennett and D. Zimmerman (Eds.), Oxford Studies in Metaphysics: Vol. 8 (pp. 1-74). Oxford: Oxford University Press.

Dretske, F. (1995). Naturalizing the mind. Cambridge, MA: MIT Press.

Earman, J. (1991). Kant, Incongruous Counterparts, and the Nature of Space and Space-Time. In J. van Cleve (ed.) The Philosophy of Left and Right (pp. 131-150). Dordrecht, Kluwer.

Evans, G. (1985). Molyneux's question. In A. Phillips (Ed.) Gareth Evans Collected Papers (pp. 364-399), Oxford: Clarendon Press.

Gardner, M. (1964). The Ambidextrous Universe. New York: Basic Books.

Hoefer, C. (2000). Kant's hands and Earman's pions: Chirality arguments for substantival space. International Studies in the Philosophy of Science 14, 237-56. doi: $10.1080 / 026985900437755$

Horgan, T. \& Tienson, J. (2002). The intentionality of phenomenology and the phenomenology of intentionality. In D.J. Chalmers (Ed.), Philosophy of Mind: Classical and Contemporary Readings (pp. 520-33). Oxford: Oxford University Press..

Jackson, F. (1982). Epiphenomenal qualia. Philosophical Quarterly, 32, 127-136. doi: $10.2307 / 2960077$ 
Johnston, M. (1996). Is the External World Invisible? Philosophical Issues, 7, 185-198. doi:

$10.2307 / 1522904$

Kant, I. (1768/1991). On the First Ground of the Distinction of Regions in Space. In J. van Cleve (Ed.) The Philosophy of Left and Right (pp. 27-34). Dordrecht, Kluwer.

Kriegel, U. (2009). Subjective Consciousness: A Self-Representational Theory. Oxford: Oxford University Press.

Kripke, S. (1980) Naming and Necessity. Cambridge, MA: Harvard University Press.

Lee, G. (2006). The experience of left and right. In T.S. Gendler \& J. Hawthorne (Eds.), Perceptual experience (pp. 291-315). Oxford: Oxford University Press.

Lewis, D. (1986). On the Plurality of Worlds. Wiley-Blackwell.

Lycan, W. (1996). Consciousness and experience. Cambridge, MA: MIT Press.

Mehta, N. (2012). Exploring Subjective Representationalism. Pacific Philosophical Quarterly, 93, 570-594. doi: 10.1111/j.1468-0114.2012.01439.x

Parsons, J. (ms). The shapes of incongruent counterparts. https://philpapers.org/archive/ PARTSO-2.pdf

Pautz, A. (2010). Do theories of consciousness rest on a mistake? Philosophical Issues, 20, 333-367. doi: 10.1111/j.1533-6077.2010.00189.x

Pautz, A. (2011). Can disjunctivists explain our access to the external world? Philosophical Issues, 21, 384-433. doi: 10.1111/j.1533-6077.2011.00209.x

Pautz, A. (2013). The real trouble with phenomenal externalism: new empirical evidence for a brain-based theory of consciousness. In R. Brown (Ed.), Consciousness Inside and Out: Phenomenology, Neuroscience, and the Nature of Experience (pp. 237-98). Springer: 237-298. 
Pautz, A. (ms) Perception. New York: Routledge.

Pooley, O. (2003). Handedness, parity violation, and the reality of space. In K. Brading \& E. Castellani, (Eds.) Symmetries in Physics: Philosophical Reflections (pp. 250-80). Cambridge University Press.

Prinz, J. (2006). Beyond appearances: the content of sensation and perception. In T. Gendler \& J. Hawthorne (Eds.), Perceptual Experience (pp. 434-60). Oxford University Press.

Prosser, S. (2011). Affordances and Phenomenal Character in Spatial Perception. Philosophical Review, 120, 475-513. doi: 10.1215/00318108-1334469

Schellenberg, S. (2011). Perceptual content defended. Nô̂s, 45, 714-750. doi: 10.1111/j.14680068.2010.00791.x

Schwitzgebel, E. (2019). Kant meets cyberpunk. Disputatio. Advance online publication. doi: 10.2478/disp-2019-0006

Shoemaker, S. (1994). Phenomenal character. Noûs, 28, 21-38. doi: 10.2307/2215918

Shoemaker, S. (2001). Introspection and phenomenal character. Philosophical Topics, 28, 24773. doi: $10.5840 /$ philtopics 20002825

Simon, J. (forthcoming) Experiencing Left and Right in a Non-Orientable World. Analytic Philosophy.

Speaks, J. (2015). The phenomenal and the representational. Oxford: Oxford University Press. Thompson, B. (2009). Senses for senses. Australasian Journal of Philosophy, 87, 99-117. doi: $10.1080 / 00048400802215471$

Thompson, B. (2010). The spatial content of experience. Philosophy and Phenomenological Research, 81, 146-184. doi: 10.1111/j.1933-1592.2010.00359.x 
Tye, M. (1995). Ten Problems of Consciousness: A Representational Theory of the Phenomenal Mind. Cambridge, MA: MIT Press.

van Cleve, J. (1987). Right, left, and the fourth dimension. Philosophical Review, 96, 33-68. doi: $10.2307 / 2185329$

Yli-Vakkuri, J. \& Hawthorne, J. (2018). Narrow Content. Oxford: Oxford University Press. 